Pacific Journal of Mathematics

ABELIAN OBJECTS

March 1967 


\section{ABELIAN OBJECTS}

MARY GRAY

In a category with a zero object, products and coproducts and in which the map

$$
A+B \stackrel{\left(\begin{array}{ll}
1 & 0 \\
0 & 1
\end{array}\right)}{\longrightarrow} A \times B
$$

is an epimorphism, we define abelian objects. We show that the product of abelian objects is also a coproduct for the subcategory consisting of all the abelian objects. Moreover, we prove that abelian objects constitute abelian subcategories of certain not-necessarily abelian categories, thus obtaining a generalization of the subcategory of the category of groups consisting of all abelian groups.

2. Definition and properties of Abelian objects. The direct product is not a coproduct in the category of groups as it is in the category of abelian groups. What is lacking is a canonical map from the product, i.e., the sum map of abelian groups; in particular, we need a map $A \times A \rightarrow A$ which when composed with $(1,0)$ or $(0,1)$ is the identity on $A$. For abelian groups this is the map $\left(1_{A}+1_{A}\right)$ (where $(a, b)(f+g)=a f+b g)$. On the other hand if such a map $x$ exists, then for $a, b \in A$, since $(0, a)+(b, 0)=((0+b),(a+0)), a+b=$ $((0, a)+(b, 0)) x=((0+b),(a+0)) x=b+a$ since $(1,0) x=(0,1) x=1_{A}$, i.e., $A$ is abelian.

This suggests that if we consider only objects where there is always a unique morphism from the product of the object with itself to either component which composes with either $(1,0)$ or $(0,1)$ to give the identity, we should get a generalization of abelian groups, provided the original category has certain properties which the category of groups has. Isbell [3] has also considered the existence of this map.

Let $\mathscr{C}$ be a category with a zero object, products and coproducts and in which the map

$$
A_{1}+A_{2} \stackrel{\left(\begin{array}{ll}
1 & 0 \\
0 & 1
\end{array}\right)}{\longrightarrow} A_{1} \times A_{2}
$$

is an epimorphism for each $A_{1}, A_{2} \in \mathscr{C}$. We assume that all categories considered are sufficiently small that the (representatives of) subobjects (and quotient objects) of a given object form a set.

Definition. Let $\mathscr{A}$ be the full subcategory of $\mathscr{C}$ determined by those $A \in \mathscr{C}$ which have a morphism $j$ from $A \times A \rightarrow A$ such that $(1,0) j=(0,1) j=1_{A}$. We call the objects of abelian objects. 
THEOREM 1. The product of abelian objects is abelian.

Proof. Suppose $A_{1} \times A_{2}$ is the product of abelian objects $A_{i}$ with projection maps $p_{i}, i=1,2$. We form the following products:

$$
\begin{aligned}
&\left(A_{1} \times A_{2}\right)_{k} \longrightarrow \\
&\left.\left(A_{i}\right)_{k} \longrightarrow A_{1} \times A_{2}\right) \times\left(A_{1} \times A_{2}\right) \stackrel{p_{i}^{\prime}}{\longrightarrow}\left(A_{1} \times A_{i} \stackrel{p_{i}^{j}}{\longrightarrow}\left(A_{i}\right)^{j}\right)_{i} \\
& A_{k} \times A_{k} \longrightarrow\left(A_{1} \times A_{1}\right) \times\left(A_{2} \times A_{2}\right) \stackrel{p_{i}^{\prime \prime}}{\longrightarrow} A_{i} \times A_{i}
\end{aligned}
$$

$i=1,2, j=1,2, k=1,2$, and we use the symbol $A_{k} \rightarrow A_{1} \times A_{2}$ to mean the map $\left(1_{A_{1}}, 0\right)$ for $k=1,\left(0,1_{A_{2}}\right)$ for $k=2$. Then we have

$$
z_{i}=\left(p_{1}^{\prime} p_{i}, p_{2}^{\prime} p_{i}\right):\left(A_{1} \times A_{2}\right) \times\left(A_{1} \times A_{2}\right) \longrightarrow A_{i} \times A_{i}
$$

so that

$$
\begin{aligned}
\left(A_{1} \times A_{2}\right)_{k} & \longrightarrow\left(A_{1} \times A_{2}\right) \times\left(A_{1} \times A_{2}\right) \stackrel{z_{i}}{\longrightarrow} A_{i} \times A_{i} \stackrel{p_{i}^{j}}{\longrightarrow}\left(A_{i}\right)^{j} \\
& =\left(A_{1} \times A_{2}\right)_{k} \longrightarrow\left(A_{1} \times A_{2}\right) \times\left(A_{1} \times A_{2}\right) \stackrel{p_{j}^{\prime}}{\longrightarrow}\left(A_{1} \times A_{2}\right)_{j} \stackrel{p_{i}}{\longrightarrow} A_{i}
\end{aligned}
$$

(by definition of $z_{i}$ ) and this is equal to

$$
\left(A_{1} \times A_{2}\right)_{k} \stackrel{p_{i}}{\longrightarrow}\left(A_{i}\right)^{k} \longrightarrow A_{i} \times A_{i} \stackrel{p_{i}^{j}}{\longrightarrow}\left(A_{i}\right)^{j}
$$

since both are projections or zero depending upon whether or not $j=k$. Moreover, the $p_{i}^{j}$ are right cancellable since the results hold for both $j=1, j=2$, and $A_{i} \times A_{i}$ is a product. Since the $A_{i}$ are abelian, there is a morphism $x_{i}: A_{i} \times A_{i} \rightarrow A_{i}$ such that $\left(1_{A_{i}}, 0\right) x_{i}=$ $\left(0,1_{A_{i}}\right) x_{i}=1_{A_{i}}$. So we define $y=\left(p_{1}^{\prime \prime} x_{1}, p_{2}^{\prime \prime} x_{2}\right), z=\left(z_{1}, z_{2}\right)$. Then we have

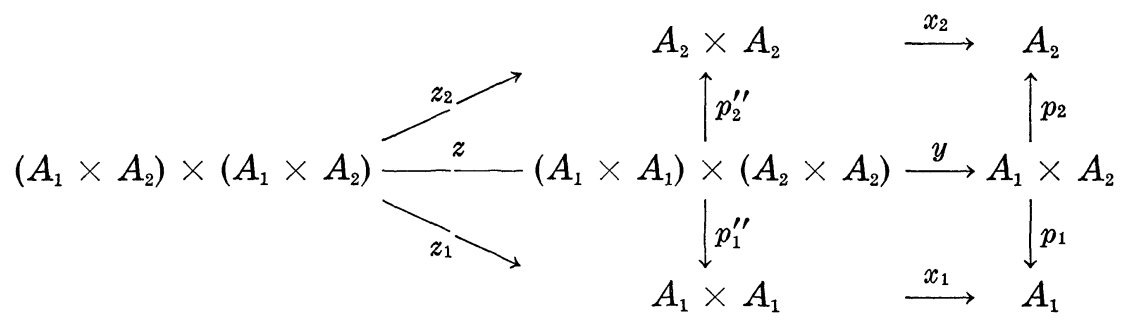

commutative from the definitions of $z_{i}, y$ and $z$. But by the above 


$$
\begin{aligned}
\left(A_{1} \times A_{2}\right)_{k} \longrightarrow & \left(A_{1} \times A_{2}\right) \times\left(A_{1} \times A_{2}\right) \stackrel{z}{\longrightarrow}\left(A_{1} \times A_{1}\right) \times\left(A_{2} \times A_{2}\right) \\
& \stackrel{y}{\longrightarrow}\left(A_{1} \times A_{2}\right) \stackrel{p_{i}}{\longrightarrow} A_{i} \\
= & \left(A_{1} \times A_{2}\right)_{k} \longrightarrow\left(A_{1} \times A_{2}\right) \times\left(A_{1} \times A_{2}\right) \stackrel{z_{i}}{\longrightarrow} A_{i} \times A_{i} \stackrel{x_{i}}{\longrightarrow} A_{i} \\
= & \left(A_{1} \times A_{2}\right)_{k} \longrightarrow\left(A_{i}\right)^{k} \longrightarrow A_{i} \times A_{i} \stackrel{x_{i}}{\longrightarrow} A_{i}=A_{1} \times A_{2} \stackrel{p_{i}}{\longrightarrow} A_{i} \\
= & A_{1} \times A_{2} \stackrel{1}{\longrightarrow} A_{1} \times A_{2} \stackrel{p_{i}}{\longrightarrow} A_{i},
\end{aligned}
$$

$i=1,2, k=1,2$. Now the $p_{i}$ are right cancellable since the equations hold for $i=1,2$. Hence $\left(1_{A_{1} \times A_{2}}, 0\right) z y=1_{A_{1} \times A_{2}}$ and $\left(0,1_{A_{1} \times A_{2}}\right) z y=1_{A_{1} \times A_{2}}$, i.e., $z y$ is the desired map.

Proposition. $X$ is abelian if and only if every morphism $\left(\begin{array}{c}f \\ g\end{array}\right): A_{1}+A_{2} \rightarrow X$ can be factored through $A_{1} \times A_{2} . \quad\left(A_{1}, A_{2}\right.$ not necessarily abelian)

Proof. If $X$ is abelian we have $\left(\begin{array}{l}f \\ g\end{array}\right)=\left(\begin{array}{ll}1 & 0 \\ 0 & 1\end{array}\right)(f, g) x$, where $X \times X \stackrel{x}{\longrightarrow} X$ is the abelianess map. If $X$ has the given property, it is abelian by virtue of factorization of $\left(\begin{array}{l}1 \\ 1\end{array}\right)$.

THEOREM 2. The product of abelian objects in $\mathscr{C}$ is also their coproduct in the subcategory of abelian objects.

Proof. If $A_{1}$ and $A_{2}$ are abelian, so is their product and since $\left(\begin{array}{ll}1 & 0 \\ 0 & 1\end{array}\right)$ is an epimorphism the factorization of the proposition above is unique.

3. Abelian subcategories. We now define a type of category in which it will be shown that the abelian objects form an abelian subcategory.

Definition. The image of a map $A \rightarrow B$ is the smallest subobject of $B$ such that $A \rightarrow B$ factors through the representative monomorphisms.

We define coimage dually.

Definition. Let $\mathscr{S}$ be a category with a zero object, products and coproducts, satisfying the following conditions:

(1) If $K \rightarrow A$ is a kernel and $A \rightarrow B$ is an epimorphism, then 
image $(K \rightarrow B)$ is a kernel.

(2) Any morphism of $\mathscr{S}$ may be factored into (representatives of) its coimage followed by its image.

(3) Every epimorphism is a cokernel.

Then $\mathscr{S}$ is called a nearly abelian category.

Clearly the category of groups and group homomorphisms satisfies these conditions.

THEOREM 3. Let $\mathscr{S}$ be a nearly abelian category. The subcategory $\mathscr{A}$ of abelian objects of $\mathscr{S}$ is an abelian category.

Proof. $A$ zero object is clearly abelian.

Products and coproducts are abelian by Theorems 1 and 2 and the following lemma:

LEMMA 0 . In a category $\mathscr{C}$ with zero object, products, coproducts, and satisfying conditions (2) and (3).

$$
A_{1}+A_{2} \stackrel{\left(\begin{array}{ll}
1 & 0 \\
0 & 1
\end{array}\right)}{\longrightarrow} A_{1} \times A_{2}
$$

is an epimorphism, for each $A_{1}, A_{2} \in \mathscr{C}$.

We first prove

Lemma 1. If $f: A \rightarrow B$ and $g: B \rightarrow C$ are such that $g$ and $f g$ have images, then the image of $f g$ is contained in the image of $g$.

Proof. Let $I \rightarrow C$ be the image of $g$. Then $A \rightarrow B \rightarrow I \rightarrow C=$ $A \rightarrow B \rightarrow C$ so that $I \rightarrow C$ contains the image of $f g$.

LEMMA 2. In a category $\mathscr{C}$ with coproducts and images the subobjects of a given object form a complete lattice.

Proof. Let $\left\{s_{j}: A_{j} \rightarrow A \mid j \in J\right\}$ represent an arbitrary set of subobjects of $A \in \mathscr{C}$. Let $\left\{u_{j}: A_{j} \rightarrow \Sigma A_{j} \mid j \in J\right\}$ be the coproduct of the $A_{j}$. Let $u$ be the unique morphism $\Sigma A_{j} \rightarrow A$ whose composition with $u_{j}$ is $s_{j}$ for each $j$. Let $I \rightarrow A$ be the image of $u$. Then we have

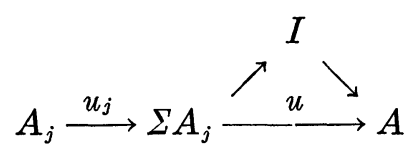

so that 


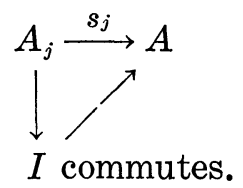

$A_{j} \rightarrow I$ is a monomorphism since $s_{j}$ is. Hence $I \rightarrow A$ is an upper bound. Suppose $s^{\prime}: A^{\prime} \rightarrow A$ is an upper bound for the $s_{j}$. Let $s_{j}^{\prime}$ be such that

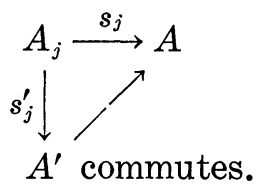

Let $v$ be the unique morphism $\Sigma A_{j} \rightarrow A^{\prime}$ whose composition with $u_{j}$ is $s_{j}^{\prime}$ for each $j$. Then we have $u_{j} v s^{\prime}=u_{j} u$; therefore $v s^{\prime}=u$ by definition of coproduct. Hence the image of $u=$ the image of $v s^{\prime}$ is contained in $s^{\prime}$ by the preceding lemma. Thus the image of $u$ is the l.u.b.

Let $\left\{s_{k}^{\prime}: A_{k}^{\prime} \rightarrow A \mid k \in K\right\}$ be the set of monomorphisms $s^{\prime}: A^{\prime} \rightarrow A$ with $s^{\prime}$ contained in $s_{j}$ for all $j \in J$. Then there exists $s^{\prime \prime}$, the l.u.b. of $\left\{s_{k}^{\prime} \mid k \in K\right\}$ (as constructed above), and $s^{\prime \prime}$ is the g.l.b. of $\left\{s_{j} \mid j \in J\right.$ ).

Proof of Lemma 0 . We have

$$
A_{1} \stackrel{u_{1}}{\longrightarrow} A_{1}+A_{2} \stackrel{\left(\begin{array}{ll}
1 & 0 \\
0 & 1
\end{array}\right)}{\longrightarrow} A_{1} \times A_{2} \stackrel{p_{1}}{\longrightarrow} A_{1}=A_{1} \stackrel{(1,0)}{\longrightarrow} A_{1} \times A_{2} \stackrel{p_{1}}{\longrightarrow} A_{1}
$$

and similarly for $p_{2}$. Then $u_{1}\left(\begin{array}{ll}1 & 0 \\ 0 & 1\end{array}\right)=(1,0)$ since the equations hold for both projections. Similarly $u_{2}\left(\begin{array}{ll}1 & 0 \\ 0 & 1\end{array}\right)=(0,1)$. By the construction of Lemma 2 , the l.u.b. of $(1,0)$ and $(0,1)$ is image $\left(A_{1}+A_{2} \rightarrow A_{1} \times A_{2}\right)$. Hence by definition of product, domain image $\left(A_{1}+A_{2} \rightarrow A_{1} \times A_{2}\right)$ is (isomorphic to) $A_{1} \times A_{2}$. Thus

$$
\begin{aligned}
A_{1}+ & A_{2} \rightarrow A_{1} \times A_{2} \\
& =\text { coimage }\left(A_{1}+A_{2} \longrightarrow A_{1} \times A_{2}\right)\left(A_{1} \times A_{2} \longrightarrow A_{1} \times A_{2}\right) \\
& =\left(A_{1}+A_{2} \stackrel{\left(\begin{array}{ll}
1 & 0 \\
0 & 1
\end{array}\right)}{\longrightarrow} A_{1} \times A_{2}\right)\left(A_{1} \times A_{2} \longrightarrow A_{1} \times A_{2}\right)
\end{aligned}
$$

and since $A_{1} \times A_{2} \rightarrow A_{1} \times A_{2}$ is right cancellable,

$$
\left(\begin{array}{ll}
1 & 0 \\
0 & 1
\end{array}\right)=\text { coimage }\left(A_{1}+A_{2} \longrightarrow A_{1} \times A_{2}\right)
$$


and hence it is an epimorphism.

It now remains to show only that every morphism has both a kernel and a cokernel and that every monomorphism is a kernel and that every epimorphism is a cokernel.

LEMMA 2*. In a category with products and coimages the quotient objects of a given object form a complete lattice.

Proof. The proof is dual to that of Lemma 2.

LEMma 3. If every morphism of a category $\mathscr{C}$ with a zero object and coproducts (products) can be factored into an epimorphism followed by a monomorphism, then every morphism has a kernel (cokernel).

Proof. We prove the coproducts and kernels case; the other proceeds dually. Let $A \rightarrow B$ be a morphism of $\mathscr{C}$. Consider the coproduct $\Sigma A_{j}$ of all subobjects of $A$ such that $A_{j} \rightarrow A \rightarrow B=0$. Then $\Sigma A_{j} \rightarrow A \rightarrow B=0$ by definition of coproduct so let $\Sigma A_{j} \rightarrow A=\Sigma A_{j} \rightarrow$ $I \rightarrow A, \Sigma A_{j} \rightarrow I$ an epimorphism, $I \rightarrow A$ a monomorphism, i.e., we have

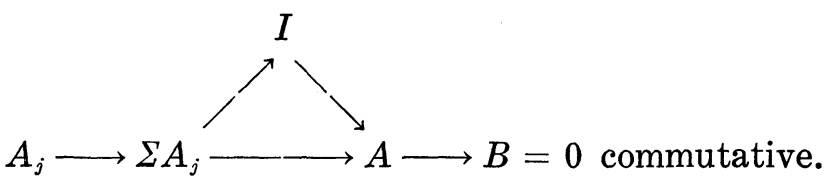

Then $\Sigma A_{j} \rightarrow I \rightarrow A \rightarrow B=0$ and since $\Sigma A_{j} \rightarrow I$ is an epimorphism, $I \rightarrow A \rightarrow B=0$. Moreover, $I \rightarrow A$ is an upper bound for the $A_{j}$, for there is a map $A_{j} \rightarrow I=A_{j} \rightarrow \Sigma A_{j} \rightarrow I$ such that

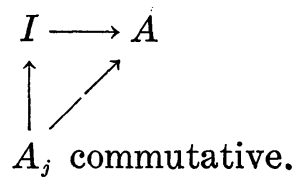

for each $A_{j}$. Hence $I \rightarrow A$ is the desired kernel.

LEMMA 4. In a category $\mathscr{C}$ with kernels and cokernels in which every epimorphism is a cokernel, if $A \rightarrow B$ factors through an epimorphism $A \rightarrow C$ and a monomorphism $C \rightarrow B$, this factorization is unique up to equivalence.

Proof. Suppose $A \rightarrow C^{\prime} \rightarrow B$ and $A \rightarrow C \rightarrow B$ are two factorizations of $A \rightarrow B$ into an epimorphism followed by a monomorphism. Let $K \rightarrow A$ be the kernel of $A \rightarrow C$; then $A \rightarrow C$ is the cokernel of $K \rightarrow A$ and similarly for $K^{\prime} \rightarrow A$ and $A \rightarrow C^{\prime}$. Then $K \rightarrow A \rightarrow C^{\prime} \rightarrow B=0$ 
and $K \rightarrow A \rightarrow C^{\prime}=0$ since $C^{\prime} \rightarrow B$ is right cancellable. Hence $K \rightarrow A$ is contained in $K^{\prime} \rightarrow A$ and hence $A \rightarrow C$ contains $A \rightarrow C^{\prime}$. Similarly $A \rightarrow C^{\prime}$ contains $A \rightarrow C$. Now we have

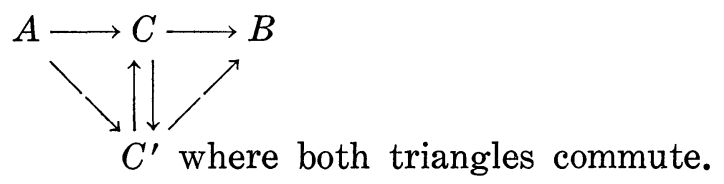

Since $A \rightarrow C^{\prime}$ is an epimorphism, $C^{\prime} \rightarrow C \rightarrow B=C^{\prime} \rightarrow B$ and similarly $C \rightarrow C^{\prime} \rightarrow B=C \rightarrow B$. Hence $C^{\prime} \rightarrow B$ and $C \rightarrow B$ are also equivalent.

Lemma 5. In a category as in Lemma 0 if $f: A \rightarrow B$ is an epimorphism and $g: B \rightarrow C$, then image of $f g=$ image of $g$.

Proof. Let $I \rightarrow C$ be the image of $B \rightarrow C$. Then $A \rightarrow I$ is the composition of epimorphisms

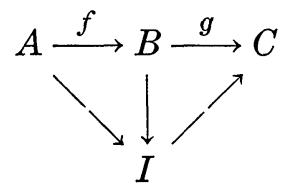

and hence an epimorphism. Thus by Lemma 4 it is the coimage of $A \rightarrow C$ and $I \rightarrow C$ is the image of $A \rightarrow C$.

Lemma 6. In a category such as in Lemma 0, if $m_{1}: A_{1} \rightarrow A$, $m_{2}: A_{2} \rightarrow A$ are monomorphisms and $f: A \rightarrow C$, then

image ((l.u.b. $\left.\left.\left\{m_{1}, m_{2}\right\}\right) f\right)=$ image (l.u.b. \{image $m_{1} f$, image $\left.\left.m_{2} f\right\}\right)$.

Proof. Let $u_{i}: A_{i} \rightarrow A_{1}+A_{2}, u_{i}^{\prime}: A_{i}^{\prime} \rightarrow A_{1}^{\prime}+A_{2}^{\prime}$, where $A_{i}^{\prime} \rightarrow C$ is the image of $m_{i} f$. Then we have

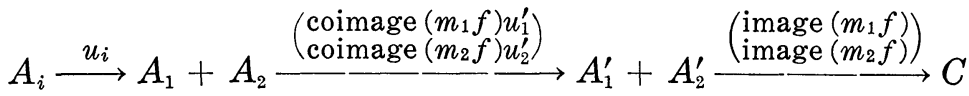

$$
\begin{aligned}
& =A_{i} \stackrel{\text { coimage }\left(m_{i} f\right)}{\longrightarrow} A_{i}^{\prime} \longrightarrow A_{1}^{\prime}+A_{2}^{\prime} \stackrel{\left(\begin{array}{l}
\text { image }\left(m_{1} f\right) \\
\text { image }\left(m_{2} f\right)
\end{array}\right)}{\longrightarrow} C \\
& =A_{i} \stackrel{\text { coimage }\left(m_{i} f\right)}{\longrightarrow} A_{i}^{\prime} \stackrel{\text { (image } \left.m_{i} f\right)}{\longrightarrow} C \\
& =A_{i} \stackrel{u_{i}}{\longrightarrow} A_{1}+A_{2} \stackrel{\left(\begin{array}{l}
m_{1} \\
m_{2}
\end{array}\right)}{\longrightarrow} A \stackrel{f}{\longrightarrow} C \text {. }
\end{aligned}
$$

Since these equations hold for $u_{1}$ and $u_{2},\left(\begin{array}{l}\text { coimage }\left(m_{1} f\right) u_{1}^{\prime} \\ \text { coimage }\left(m_{2} f\right) u_{2}^{\prime}\end{array}\right)\left(\begin{array}{l}\text { image }\left(m_{1} f\right) \\ \text { image }\left(m_{2} f\right)\end{array}\right)=$ $\left(\begin{array}{l}m_{1} \\ m_{2}\end{array}\right) f$. Then image $\left(A_{i} \stackrel{u_{i}}{\longrightarrow} A_{1}+A_{2} \rightarrow A_{1}^{\prime}+A_{2}^{\prime}\right)$ is contained in the 
image of $A_{1}+A_{2} \rightarrow A_{1}^{\prime}+A_{2}^{\prime}$. But by the factorization above and the fact that $A_{1}+A_{2}$ is a coproduct, the image of $A_{i} \rightarrow A_{1}+A_{2} \rightarrow A_{1}^{\prime}+A_{2}^{\prime}$ is $u_{i}^{\prime}$. Thus since the l.u.b. of the $u_{i}^{\prime}$ 's is $A_{1}^{\prime}+A_{2}^{\prime} \rightarrow A_{1}^{\prime}+A_{2}^{\prime}$, this identity is the image of $A_{1}+A_{2} \rightarrow A_{1}^{\prime}+A_{2}^{\prime}$ and $A_{1}+A_{2} \rightarrow A_{1}^{\prime}+A_{2}^{\prime}$ is its own coimage and hence an epimorpism. Then the image of $\left(\begin{array}{l}\text { coimage }\left(m_{1} f\right) u_{1}^{\prime} \\ \text { coimage }\left(m_{2} f\right) u_{2}^{\prime}\end{array}\right)\left(\begin{array}{l}\text { image }\left(m_{1} f\right) \\ \text { image }\left(m_{2} f\right)\end{array}\right)$ is the image of the second map by Lemma 5.

Also we have

$$
\text { image }\left[\left(\begin{array}{l}
m_{1} \\
m_{2}
\end{array}\right) f\right]=\text { image }\left[\left(\operatorname{image}\left(\begin{array}{l}
m_{1} \\
m_{2}
\end{array}\right)\right) f\right]
$$

since the coimage of $\left(\begin{array}{l}m_{1} \\ m_{2}\end{array}\right)$ is an epimorphism. We have

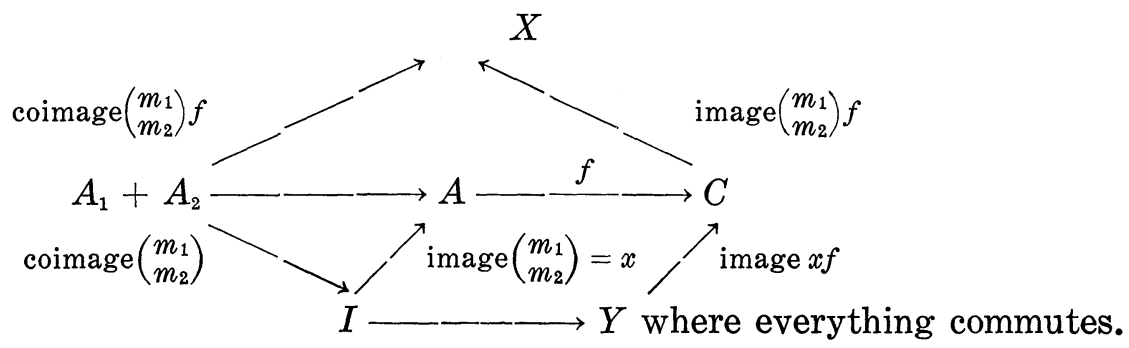

Then

$$
\begin{aligned}
\text { image } & {\left[\left(\text { image }\left(\begin{array}{c}
m_{1} \\
m_{2}
\end{array}\right)\right) f\right]=\text { image }\left(\left(\text { l.u.b. }\left\{m_{1}, m_{2}\right\}\right) f\right) } \\
& \left.=\text { image (l.u.b. }\left\{\text { image }\left(m_{1} f\right), \text { image }\left(m_{2} f\right)\right\}\right)
\end{aligned}
$$

since we get from the above that

$$
\begin{aligned}
\text { image } & {\left[\left(\begin{array}{l}
\text { coimage }\left(m_{1} f\right) u_{1}^{\prime} \\
\text { coimage }\left(m_{2} f\right) u_{2}^{\prime}
\end{array}\right)\left(\begin{array}{l}
\text { image }\left(m_{1} f\right) \\
\text { image }\left(m_{2} f\right)
\end{array}\right)\right]=\text { image }\left[\left(\begin{array}{l}
\text { image }\left(m_{1} f\right) \\
\text { image }\left(m_{2} f\right)
\end{array}\right)\right] } \\
& =\text { image }\left[\left(\begin{array}{l}
m_{1} \\
m_{2}
\end{array}\right) f\right]=\text { image }\left[\left(\operatorname{image}\left(\begin{array}{l}
m_{1} \\
m_{2}
\end{array}\right)\right)\right] f,
\end{aligned}
$$

which proves the lemma.

We now show that any subobject of an abelian object is an abelian object. If in particular the subobject is the kernel in $\mathscr{S}$ of a morphism of $\mathscr{A}$, then it is in $\mathscr{A}$ and clearly is the kernel in $\mathscr{A}$. Suppose $k: K \rightarrow A$ is a subobject of an abelian object $A$. Let $K \times K$ be the product of $K$ with itself, $p_{i}$ its projection morphisms, $p_{i}^{\prime}$ the projection morphisms for $A \times A$. Let $x$ be the morphism $A \times A \rightarrow A$ such that $A_{i} \rightarrow A \times A \stackrel{x}{\longrightarrow} A=1_{A}, i=1,2$. Let $y=\left(p_{1} k, p_{2} k\right)$ so that $K_{i} \rightarrow$ $K \times K \stackrel{y}{\longrightarrow} A \times A \stackrel{x}{\longrightarrow} A=k$ as in Theorem $2 . \quad K \times K \rightarrow K \times K$ is 
the l.u.b. of $K_{1} \rightarrow K \times K$ and $K_{2} \rightarrow K \times K$ so

image ((l.u.b. $\left.\left.\left\{K_{1} \longrightarrow K \times K, K_{2} \longrightarrow K \times K\right\}\right) y x\right)=$ image $y x$.

Moreover,

$$
\begin{aligned}
& \text { l.u.b. }\left\{\text { image }\left(K_{1} \longrightarrow K \times K \stackrel{y x}{\longrightarrow} A\right) \text {, image }\left(K_{2} \longrightarrow K \times K \stackrel{y x}{\longrightarrow} A\right)\right\} \\
& =\text { image } k
\end{aligned}
$$

and by Lemma 6 , image $y x=$ image $k$.

Now we let $x^{\prime}: K \times K \rightarrow K$ be the coimage of $y x$. Then $\left(1_{K}, 0\right) x^{\prime} k=$ $\left(1_{K}, 0\right)($ coimage $(y x))$ (image $\left.(y x)\right)=\left(1_{K}, 0\right) y x=k\left(1_{A}, 0\right) x=k($ by definition of $x)$ and similarly for $\left(0,1_{K}\right)$. Then $k$ is right cancellable so $\left(1_{K}, 0\right) x^{\prime}=$ $1_{K}$ and $\left(0,1_{K}\right) x^{\prime}=1_{K}$. Hence $x^{\prime}$ is the desired morphism and $K \in \mathscr{A}$.

Dually to the above, any quotient object of an abelian object is abelian, and in particular the cokernel of a morphism of $\mathscr{A}$ is in $\mathscr{A}$.

We now show that all monomorphisms of $\mathscr{A}$ are kernels. Suppose $f: A \rightarrow B$ is a monomorphism of $\mathscr{A}$. Let $B \times B \stackrel{p_{i}}{\longrightarrow} B_{i}, A \times B \stackrel{p_{1}^{\prime}}{\longrightarrow} A$, $A \times B \stackrel{p_{2}^{\prime}}{\longrightarrow} B$ be products. Then we have $\left(p_{1}^{\prime} f, p_{2}^{\prime}\right): A \times B \rightarrow B \times B$ and $A \stackrel{(1,0)}{\longrightarrow} A \times B \rightarrow B \times B=A \rightarrow B \stackrel{(1,0)}{\longrightarrow} B \times B$ since followed by either $p_{i}$ they are equal. Moreover, $B \stackrel{(0,1)}{\longrightarrow} A \times B \rightarrow B \times B=B \stackrel{(0,1)}{\longrightarrow}$ $B \times B$. Let $j$ be the morphism such that $\left(1_{B}, 0\right) j=1_{B}=\left(0,1_{B}\right) j$. Then $B \rightarrow A \times B \rightarrow B \times B \stackrel{j}{\longrightarrow} B=B \stackrel{(0,1)}{\longrightarrow} B \times B \stackrel{j}{\longrightarrow} B=1_{B}$; hence $\left(p_{1}^{\prime} f, p_{2}^{\prime}\right) j$ is an epimorphism since $1_{B}$ is. Then

$$
\begin{aligned}
& A \longrightarrow A \times B \longrightarrow B \times B \stackrel{j}{\longrightarrow} B \\
&=A \stackrel{f}{\longrightarrow} B \stackrel{(1,0)}{\longrightarrow} B \times B \stackrel{j}{\longrightarrow} B=A \stackrel{f}{\longrightarrow} B .
\end{aligned}
$$

Now $A \rightarrow A \times B$ is a kernel of $A \times B \rightarrow B$ and since $A \times B \rightarrow$ $B \times B \stackrel{j}{\longrightarrow} B$ is an epimorphism, $A \rightarrow A \times B \rightarrow B \times B \stackrel{j}{\longrightarrow} B=A \rightarrow$ $B=$ image $(A \rightarrow B)$ (since $A \rightarrow B$ is a monomorphism) is a kernel by condition (1).

If $f: A \rightarrow B$ is an epimorphism in $\mathscr{S}$ we form its kernel as above and it is the cokernel of its kernel. It remains to show that if $f$ is an epimorphism of $\mathscr{A}$, it is an epimorphism of $\mathscr{S}$.

Suppose $f: A \rightarrow B$ is an epimorphism of $\mathscr{A}$. Then suppose $B \rightarrow I$ is the cokernel of $A \rightarrow B$. Since $I$ is abelian and $A \rightarrow B$ is left cancellable in $\mathscr{A}, B \rightarrow I=0$, i.e., the cokernel of $f$ is zero. Then its kernel is the image of $f$, which is then equivalent to $B \rightarrow B$, i.e., $A \rightarrow B$ is its own coimage and hence an epimorphism.

Thus $\mathscr{A}$ is abelian, completing the proof of Theorem 3 . 
4. $H$-spaces. In the category $\mathscr{T}$ of topological spaces with base points and continuous maps taking base points into base points, we call a map $\mu: X \times X \rightarrow X$ (Cartesian product) a continuous multiplication. We denote $(a, b) \mu$ by $a b$. The correspondences $x \rightarrow a x$ and $x \rightarrow x a$ for a given $a \in X$ determine the maps $L_{a}: X \rightarrow X, R_{a}: X \rightarrow X$. A base point $a \in X$ is a homotopy unit if $a$ is idempotent and $L_{a}$ and $R_{a}$ are homotopic to the identity map relative to $a . R_{a}$ and $L_{a}$ are continuous by definition and take base points into base points since $a$ is idempotent. $X$ is an $H$-space if it has a continuous multiplication with homotopy unit.

Clearly $R_{a}$ factors through $X \times X$ (which is obviously a product in this category) as $X \stackrel{(1,0)}{\longrightarrow} X \times X \stackrel{\mu}{\longrightarrow} X$, and similarlyf or $L_{a}$. If $a$ is a homotopy unit,

$$
\begin{aligned}
& X \stackrel{(1,0)}{\longrightarrow} X \times X \stackrel{\mu}{\longrightarrow} X=R_{a} \simeq l_{X} \\
& X \stackrel{(0,1)}{\longrightarrow} X \times X \stackrel{\mu}{\longrightarrow} X=L_{a} \simeq l_{X} .
\end{aligned}
$$

Now consider the functor $\pi_{1}$ from the category $\mathscr{T}$ to the category $\mathscr{G}$ of groups and group homomorphisms which assigns to each object of $\mathscr{T}$ its fundamental group. We know that $(X \times X) \pi_{1}=$ $(X) \pi_{1} \times(X) \pi_{1}$ (group direct product) so we have

$$
(X) \pi_{1} \stackrel{(1,0) \pi_{1}}{\longrightarrow}(X) \pi_{1} \times(X) \pi_{1} \stackrel{(\mu) \pi_{1}}{\longrightarrow}(X) \pi_{1}=\left(R_{a}\right) \pi_{1}=\left(1_{X}\right) \pi_{1}
$$

(since $\left.R_{a} \simeq 1_{X}\right)=1_{(X) \pi_{1}}$. Moreover, $(1,0) \pi_{1}=\left(1_{(X) \pi_{1}}, 0\right)$ and similarly for $(0,1) \pi_{1}$ by definition of product and functor. Hence $(\mu) \pi_{1}$ is the required map in the definition of abelian objects. Thus we obtain the well-known result that the fundamental group of an $H$-space is abelian.

The author wishes to thank the referee for his many helpful suggestions.

\section{REFERENCES}

1. Peter Freyd, Abelian Categories, Harper and Row, New York, 1964.

2. S. T. Hu, Homotopy Theory, Academic Press, New York, 1959.

3. J. R. Isbell, Natural sums and Abelianizing, Pacific J. Math. 14 (1964), 1265-1282.

4. Saunders MacLane, Categorical Algebra, Pacific J. Math. 14 (1964), 1265-1282.

Received January 6, 1965.

The University of California, Berkeley

California State College, Hayward 


\section{PACIFIC JOURNAL OF MATHEMATICS}

\section{EDITORS}

\section{H. ROYDEN}

Stanford University

Stanford, California

J. P. JANS

University of Washington

Seattle, Washington 98105

\section{J. DUGUNDJI}

Department of Mathematics

Rice University

Houston, Texas 77001

RICHARD ARENS

University of California

Los Angeles, California 90024

ASSOCIATE EDITORS

E. F. BECKENBACH

B. H. NeumanN

F. WoLF

K. YosidA

\section{SUPPORTING INSTITUTIONS}

\author{
UNIVERSITY OF BRITISH COLUMBIA \\ CALIFORNIA INSTITUTE OF TECHNOLOGY \\ UNIVERSITY OF CALIFORNIA \\ MONTANA STATE UNIVERSITY \\ UNIVERSITY OF NEVADA \\ NEW MEXICO STATE UNIVERSITY \\ OREGON STATE UNIVERSITY \\ UNIVERSITY OF OREGON \\ OSAKA UNIVERSITY \\ UNIVERSITY OF SOUTHERN CALIFORNIA
}

\author{
STANFORD UNIVERSITY \\ UNIVERSITY OF TOKYO \\ UNIVERSITY OF UTAH \\ WASHINGTON STATE UNIVERSITY \\ UNIVERSITY OF WASHINGTON \\ AMERICAN MATHEMATICAL SOCIETY \\ CHEVRON RESEARCH CORPORATION \\ TRW SYSTEMS \\ NAVAL ORDNANCE TEST STATION
}




\section{Pacific Journal of Mathematics}

\section{Vol. 23, No. 1 \\ March, 1967}

M. J. C. Baker, A spherical Helly-type theorem ................... 1

Robert Morgan Brooks, On locally m-convex*-algebras.............. 5

Lindsay Nathan Childs and Frank Rimi DeMeyer, On automorphisms of separable algebras ...................................

Charles L. Fefferman, A Radon-Nikodym theorem for finitely additive set

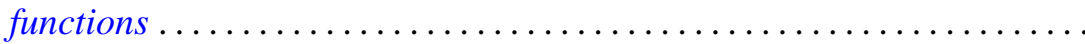

Magnus Giertz, On generalized elements with respect to linear

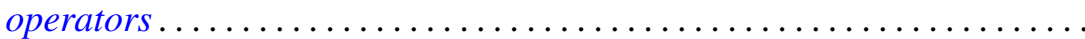

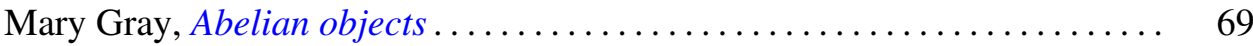

Mary Gray, Radical subcategories.............................. 79

John A. Hildebrant, On uniquely divisible semigroups on the two-cell . . . . . 91

Barry E. Johnson, AW*-algebras are $\mathrm{QW}^{*}$-algebras ............... 97

Carl W. Kohls, Decomposition spectra of rings of continuous functions . . . . 101

Calvin T. Long, Addition theorems for sets of integers .............. 107

Ralph David McWilliams, On $w^{*}$-sequential convergence and quasi-reflexivity ................................... 113

Alfred Richard Mitchell and Roger W. Mitchell, Disjoint basic

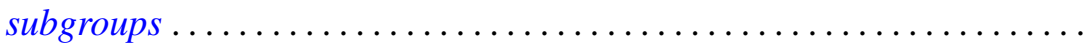

John Emanuel de Pillis, Linear transformations which preserve hermitian and positive semidefinite operators .

Qazi Ibadur Rahman and Q. G. Mohammad, Remarks on Schwarz's lemma

Neal Jules Rothman, An $L^{1}$ algebra for certain locally compact topological semigroups ...

F. Dennis Sentilles, Kernel representations of operators and their adjoints ...

D. R. Smart, Fixed points in a class of sets

K. Srinivasacharyulu, Topology of some Kähler manifolds

Francis C.Y. Tang, On uniqueness of generalized direct decompositions .

171 Albert Chapman Vosburg, On the relationship between Hausdorff dimension and metric dimension . 